\title{
Tensor Transfer Learning for Intelligence Fault Diagnosis of Bearing with Semisupervised Partial Label Learning
}

\author{
Chaofan Hu, ${ }^{1,2}$ Zhichao Zhou, ${ }^{1}$ Biao Wang, ${ }^{1}$ WeiGuang Zheng $\mathbb{D}^{1,3,4}$ and Shuilong He $\mathbb{D}^{1}$ \\ ${ }^{1}$ School of Mechanical and Electrical Engineering, Guilin 541004, China \\ ${ }^{2}$ ShenZhen KeAnDa Electronic Technology Co. Ltd., Shenzhen 518038, China \\ ${ }^{3}$ School of Mechanical and Automotive Engineering, Guangxi University of Science and Technology, Liuzhou 545616, China \\ ${ }^{4}$ Commercial Vehicle Technology Center, Dong Feng Liuzhou Automobile Co., Ltd., Liuzhou 545005, China
}

Correspondence should be addressed to WeiGuang Zheng; weiguang.zheng@foxmail.com

Received 27 October 2021; Revised 23 November 2021; Accepted 2 December 2021; Published 13 December 2021

Academic Editor: Haidong Shao

Copyright (c) 2021 Chaofan $\mathrm{Hu}$ et al. This is an open access article distributed under the Creative Commons Attribution License, which permits unrestricted use, distribution, and reproduction in any medium, provided the original work is properly cited.

\begin{abstract}
A new tensor transfer approach is proposed for rotating machinery intelligent fault diagnosis with semisupervised partial label learning in this paper. Firstly, the vibration signals are constructed as a three-way tensor via trial, condition, and channel. Secondly, for adapting the source and target domains tensor representations directly, without vectorization, the domain adaptation (DA) approach named tensor-aligned invariant subspace learning (TAISL) is first proposed for tensor representation when testing and training data are drawn from different distribution. Then, semisupervised partial label learning (SSPLL) is first introduced for tackling a problem that it is hard to label a large number of instances and there exists much data left to be unlabeled. Ultimately, the proposed method is used to identify faults. The effectiveness and feasibility of the proposed method has been thoroughly validated by transfer fault experiments. The experimental results show that the presented technique can achieve better performance.
\end{abstract}

\section{Introduction}

Fault diagnosis is a key process to ensure a reliable and costeffective performance of engineered system research. Downtime caused by failures of components such as bearing faults directly reflects on the economic viability of large systems [1-4]. Therefore, for maintaining reliability and operational safety, fault detection has attracted a lot of attention [5].

In recent years, the fault classification method has been very successful for bearing based on an assumption, which is that candidate label sets are provided for all training examples [6]. Based on this assumption, lots of effort are taken on traditional intelligent fault diagnosis approaches. Liu et al. [7] proposed a personalized diagnosis method to detect faults in a bearing based on acceleration sensors and an finite element method (FEM) simulation driving SVM. A novel supervised sparse feature extraction method is proposed for rotating machine fault diagnosis in [8]. Reference [9] proposed a novel fault diagnosis method based on local-global deep neural network algorithm. A deep learning model named renewable fusion fault diagnosis network is proposed for updating automatically as the collected fault data increases in [10]. Nowadays, various fault diagnosis methods have enriched fields of fault diagnosis. Some novel intelligent fault diagnosis techniques [11-14] are also promoted for fault diagnosis. It can be found that these approaches are applicable to vector data only. Aside from this, some tensor-based diagnosis techniques become prosperity in the fields of fault diagnosis, especially in the age of big data $[5,15,16]$.

Although the researches above realized nice performance, they may suffer the two drawbacks as follows: (1) Through the literature review, it can be seen that an important assumption in these intelligent fault diagnosis methods is that the labeled training and unlabeled testing data come from the same distribution [17]. However, assumption fails by two main reasons [18]. Firstly, labeled fault signal are hard to be obtained from some equipments. Secondly, an intelligent fault diagnosis algorithm trained with labeled data possibly fails in classifying unlabeled data when the labeled 
and unlabeled data are subject to different machines. Thus, distribution discrepancy exists between source and target domains, which causes important classification performance degenerates [17]. In order to handle with the domain distribution problem, DA techniques have been developed [19].

DA method establishes knowledge cross-domain transfer from source to target-domains via studying a domaininvariant feature subspace [17]. DA techniques have been successfully developed and applied in $[20,21]$. Reference [22] proposed a defect identification method of wind turbine blades based on defect semantic features with transfer feature extractor. Reference [23] presented a novel domain adaptation model based on geodesic flow kernel (GFK) and strengthened feature extraction and $Z$-score normalization. Aside from this, reference [24] proposed a feature-based transfer neural network to identify the health states of motor bearings and gearbox bearings. A transferable convolutional neural network (CNN) [25] is proposed for intelligent fault diagnosis of rotary machinery.

Nevertheless, it can be seen that the existing diagnosis technique of transfer learning with DA approaches focus on the vector data. Therefore, the approaches are used to establish high-dimensional data; the data must be vectorized. Aside from this, vectorization always leads to high computational complexity and so on.

For addressing these issues, a new method is used for tensor data representation. The idea of the proposed method is that an invariant tensor subspace is used for adapting the tensor representations [17].

Different from the vector subspace, the tensor subspace concludes a set of subspaces characterizing each mode separately [21]. The proposed technique realizes mode-wise partial adaptation for reducing the dimensionality issue. Therefore, a joint optimization problem is formulated by seeking such a tensor subspace and learning the alignment matrices [17]. The issue is optimized via an alternating minimization method. In cross-domain visual recognition, the TAISL has achieved great success. However, there is no reports about TAISL for rotating machinery fault classification in available references.

(2) Through the literature review, it can be also seen that existing methods often assumes that each training example is associated with a ground-truth label. Nevertheless, one can only get access to a candidate label set associated with each training example among which only one label is valid in many practical applications [6]. Therefore, partial label learning (PLL) has been proposed for dealing with this kind of training examples in [26]. The PLL has attracted increasing research attention, so extensive PLL methods have been proposed in references $[27,28]$. However, there is no reports about PLL for rotating machinery fault classification in available literatures.

A basic assumption is that all the candidate label sets are provided for training sample in the previous researches on PLL. Nevertheless, in practical applications, such assumption is difficult to hold [6]. A fault can be labeled by a candidate label set, but there still exist many faults that have actually no label information for them.

In this work, it is clear that neither PLL nor semisupervised learning (SSL) can address the issue concerned. For instance, although some examples could be very helpful, large numbers of unlabeled instances are ignored via PLL. The SSL assumes that the ground-truth single-label is accessible to each labeled training example, which is not the case in our situation.

A new method named SSPLL is introduced into the field of bearing fault diagnosis. It is critical that the candidate label sets of partial label instances are disambiguated and the dataset distribution information of unlabeled examples is used simultaneously. Particularly, the candidate label sets of partial label instances are disambiguated by an iterative label propagation step from partial label to unlabeled examples and the iterative label propagation procedure is used to distribute valid labels to unlabeled examples in proposed algorithm. Thus, a new approach is proposed for classification of bearing faults with semisupervised partial label learning based on tensor representation. The main highlights of the proposed method are generalized as follows:

(1) To deal with domain shift issue in tensor space, a novel DA method is proposed for bearing fault diagnosis based on tensor representation

(2) To adapt the source domain and target domain based on tensor representation, the tensor transfer learning is introduced

(3) To tackle a problem that it is hard to label a large number of instances and there exists much data left to be unlabeled, a new method named SSPLL is introduced to deal with this issue in intelligent fault diagnosis field

(4) To realize the process of labeling information propagation from the source domain to the target domain, a weighted graph is established in this paper

(5) To assist the iterative label propagation step, establishing four normalized weight matrix corresponding to the four phases in the label propagation step separately in this work

The remainder of this paper is structured as follows: in Section 2, the basic theory of the proposed method is described. The explored method are illustrated in Section 3. The developed method is validated in Section 4. In Section 5 , the conclusions are drawn.

\section{The Basic Theory of the Proposed Method}

In this section, the theory of semisupervised partial label learning is introduced. $\mathscr{Y} \in \mathbb{R}^{t}$ denotes the $d$-dimensional example space and $\mathscr{X}=x_{1}, x_{2}, \cdots x_{2}$ represents the label space with $\mathrm{n}$ category labels in the raw PLL. Officially, $\mathscr{C}=\left(y_{j}, H_{j}\right) \| 1 \leq j \leq m$ means the partial label training set, where $y_{j} \in \mathcal{Y}$ denotes a $d$-dimensional signature vector $\left(y_{i 1}, y_{i 2}, \cdots y_{i t}\right.$ and $H_{j} \in \mathcal{Y}$ means the associated candidate label set. Basing on a critical assumption about PLL, the real label $y_{j}$ for $\mathbf{x}_{\mathbf{j}}$ is concealed in its candidate label set $\left(y_{j} \subseteq S_{j}\right)$ and thus is incapable of being attained by the learning method [6]. 

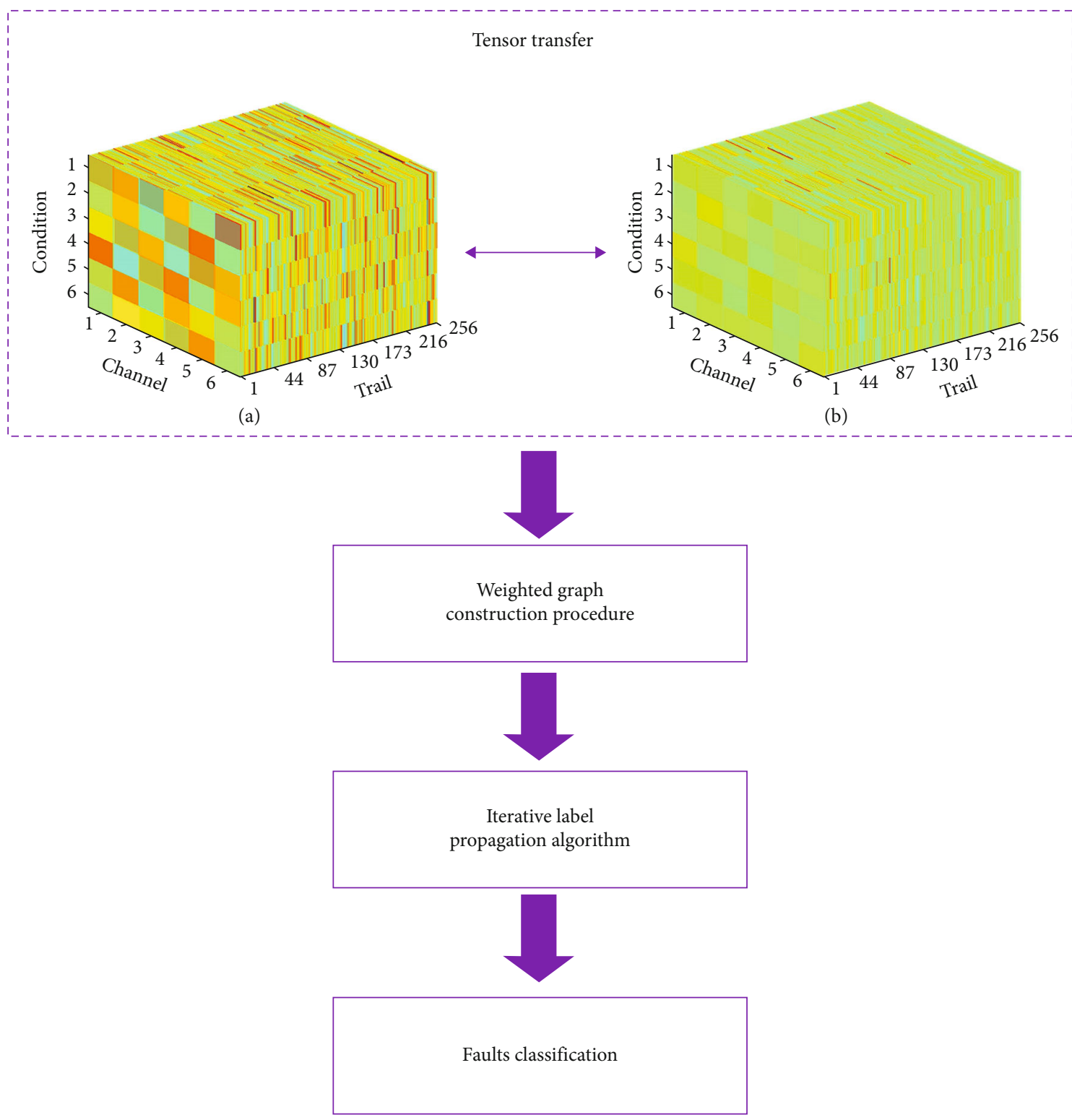

Figure 1: Framework of the proposed technique.

The training set contains partial label instances $\mathscr{D}_{p}=$ $\left(x_{j}, S_{j}\right) \| 1 \leq j \leq m$ and unlabeled examples $\mathscr{D}_{u}=x_{j} \| 1 \leq j \leq m$ in the SSPLL. According to semisupervised partial label training set $\mathscr{C}=\mathscr{D}_{p} \cup \mathscr{D}_{u}$, a recognizing model $f: \mathscr{Y} \longrightarrow \mathscr{X}$ is induced from $\mathscr{C}$ by SSPLL, $f$ predicts its label. Please refer to $[5,15,16]$ for a basic theory of the proposed method.

\section{The Proposed Method}

Framework of the proposed technique is shown in Figure 1.

3.1. TAISL. Please refer to literature [29] for a detailed discussion of the TAISL. The domain adaptation and shift based on tensor representation are illustrated in Figure 2.
3.2. A Scheme. The adapt domains problem is tackled by introducing an invariant subspace between the source $\mathcal{S}$ and the target domains $\mathscr{T}$.

SSPLL is difficult that the learning approach is needed to disambiguate the candidate label sets of partial label instances and exploit the distribution information of unlabeled data simultaneously. A simple scheme is proposed for disambiguating the candidate label sets of partial label training instances. For example, the effective single-label is found from a candidate label set. Therefore, an easy SSL issue is introduced to replace previous problem. This new problem can be tackled by learning a method.

The step of label set disambiguation and unlabeled data exploitation are completely separated in the technique above. The disambiguation accuracy are incapable of being improved via unlabeled examples. For solving this key 


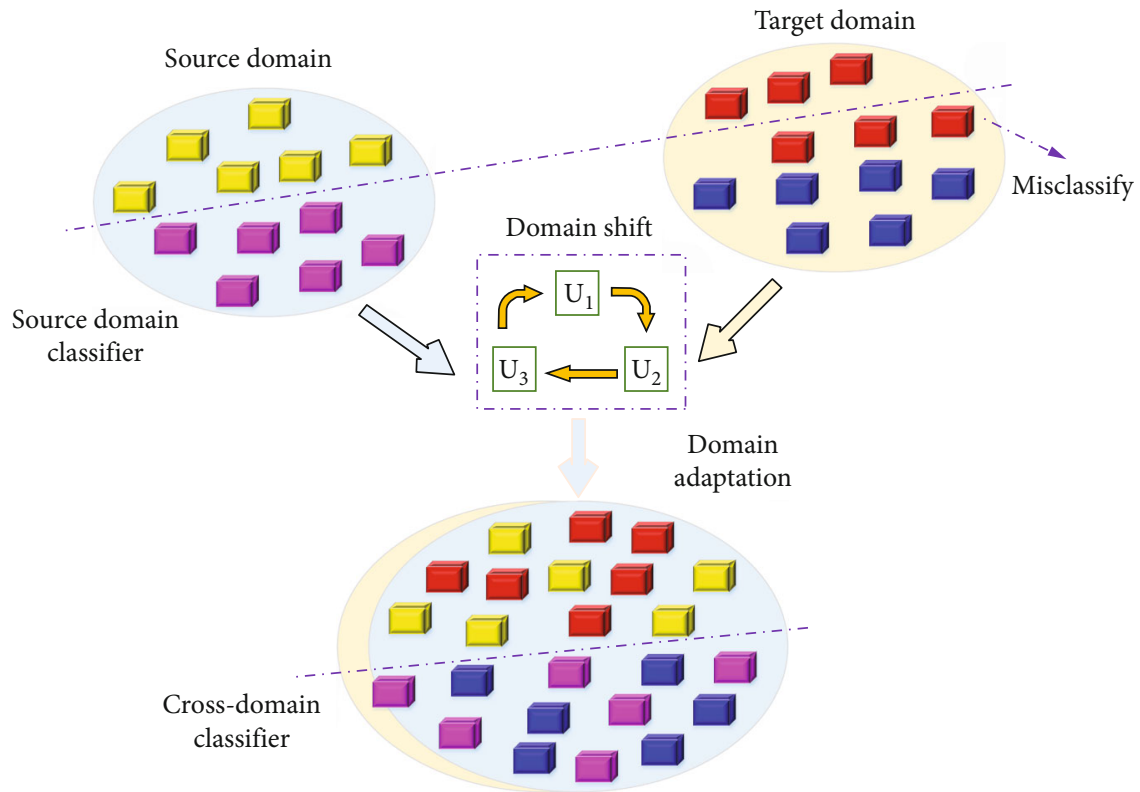

FIgURE 2: Illustrations for domain adaptation and shift based on tensor representation.

(a)

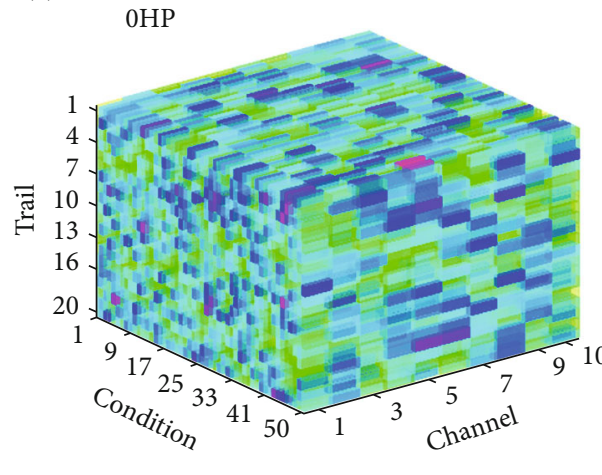

(b)

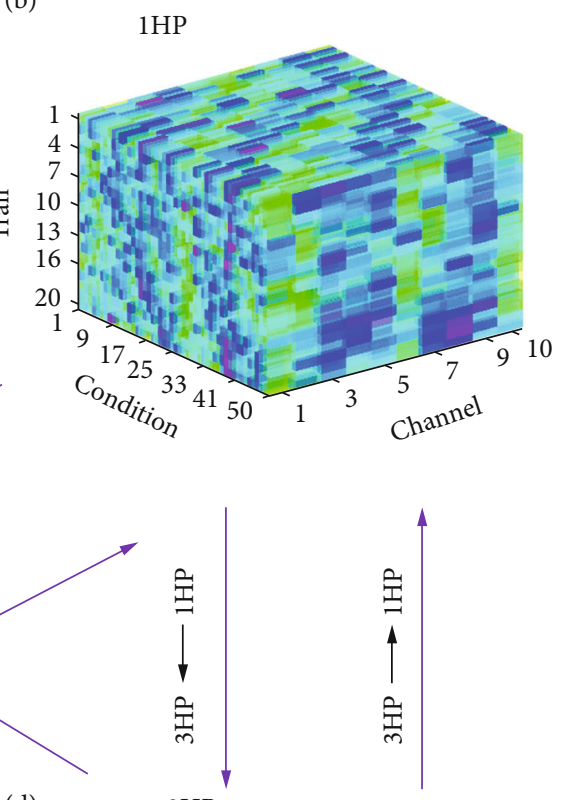

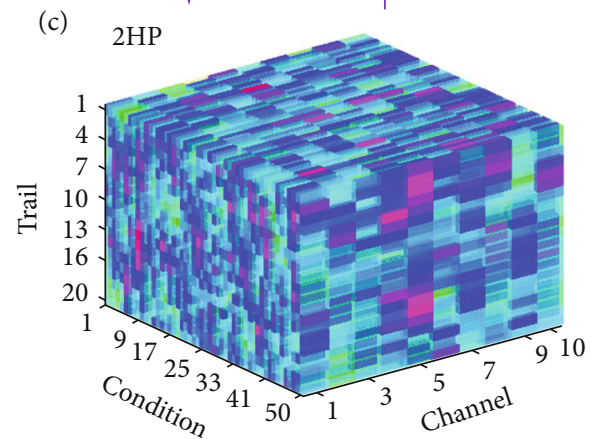
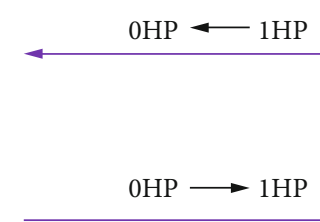


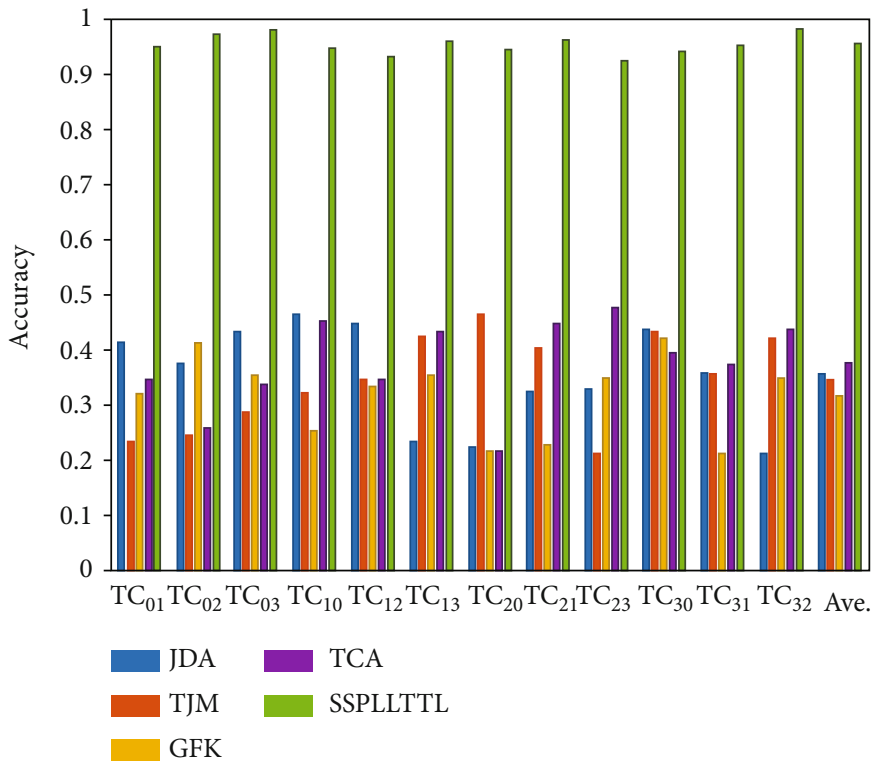

(a)

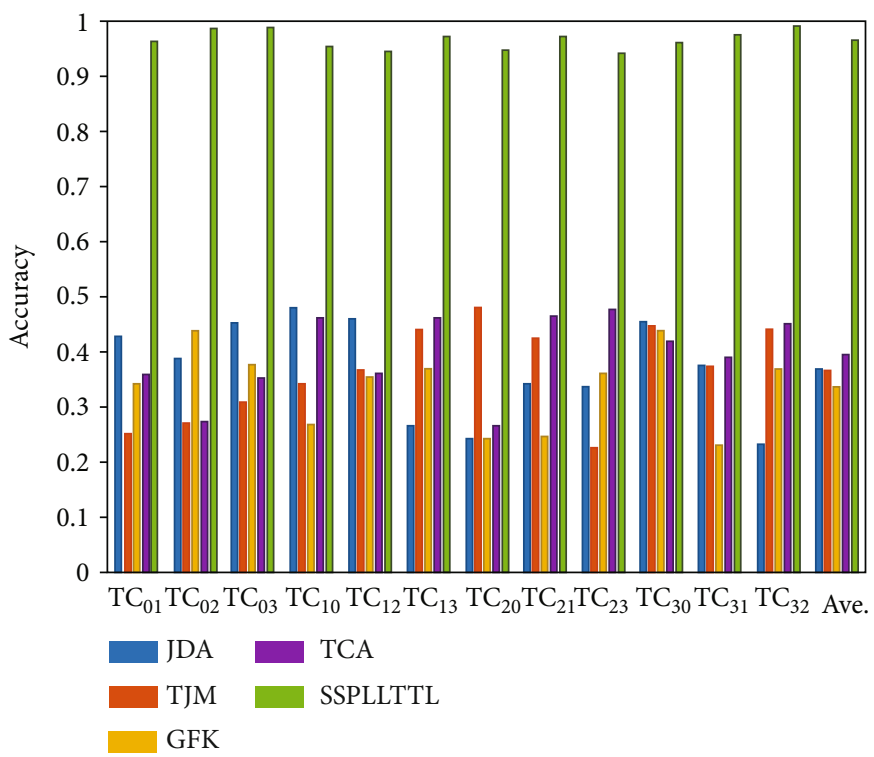

(b)

Figure 4: Continued. 


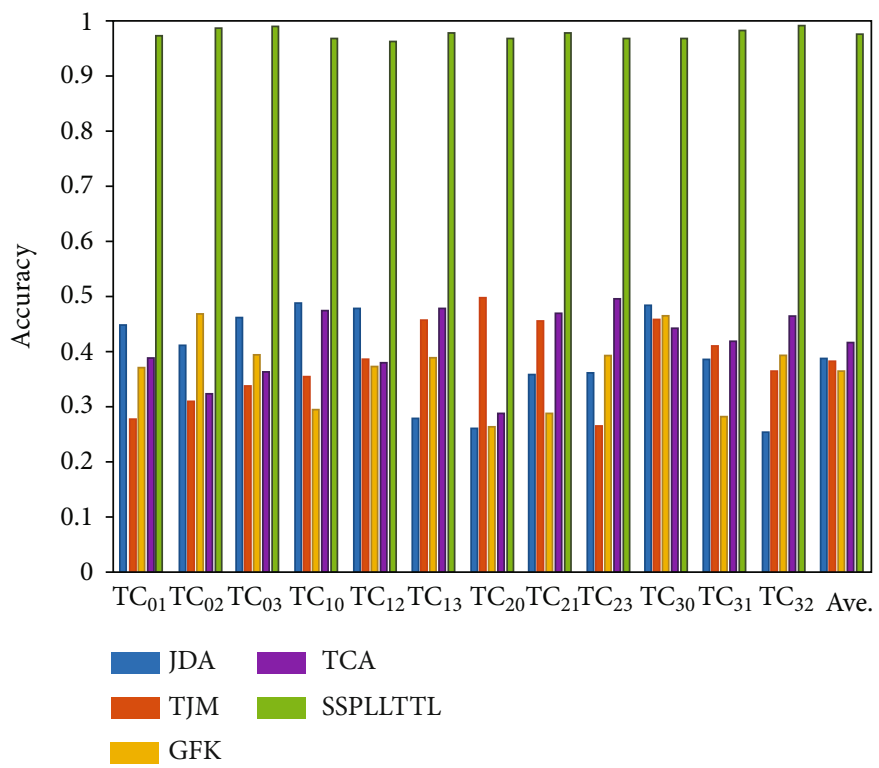

(c)

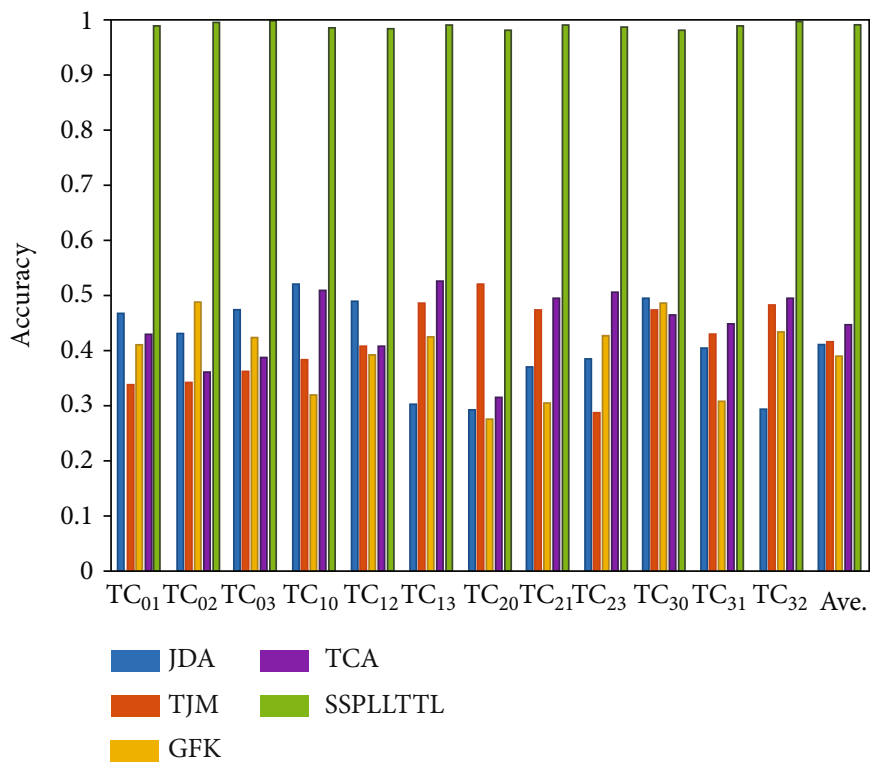

(d)

Figure 4: The partial label examples are obtained in the whole training instance by randomly sampling: (a) $p=0.1,(\mathrm{~b}) p=0.2,(\mathrm{c}) p=0.3$, and (d) $p=0.4$.

limitation, an iterative label propagation algorithm is proposed for putting partial label instances and unlabeled examples into a structure in the presented the SSPLL technique.

3.3. Weighted Graph Construction Procedure. For achieving the process of labeling information propagation between the source domain set $\mathcal{S}=y_{j} \| 1 \leq i \leq s$ and the target domain set $\mathscr{T} y_{j} \| 1 \leq j \leq t$, a weighted graph [6] $\mathbf{G}=(\mathbf{O}, \mathbf{P}, \mathbf{K})$ is established over $\mathcal{S}$ and $\mathscr{T}$, while vertex set $\mathbf{O}$ represents the examples of source training set and vertex set $\mathbf{P}$ means the examples of target training set. Edge set $\mathbf{K}$ consists of the directed edges $\mathbf{O}$ and $\mathbf{P}$. For each example $y_{j}$ in the target training set, its $k$-nearest neighbors $\mathcal{N}\left(y_{j}\right)$ in the source training set are recognized. Thus, the edges of graph $\mathbf{G}$ are set as $\mathbf{K}=x_{i} ; x_{j} \| x_{j} \in \mathcal{N}\left(x_{j}\right) ; 1 \leq j \leq t$.

According to the graph $\mathbf{G}$ established above, a $t \times s$ weight matrix $\mathbf{W}=\left[w_{(i, j)}\right]_{(t \times s)}$ is able to be specified, where $w_{(j, i)} \gg 0$ if $\left(x_{i}, x_{j}\right) \in$ Eand $w_{(j, i)}=0$ otherwise. In this paper, for capturing the little influences between examples, the weight calculation approach is proposed for applying in the IPAL method [27], which selects the weights by addressing a new optimization issue:

$$
\min _{\mathscr{W}_{j}}\left\|\mathbf{x}_{j}-\sum_{a=1}^{k} W_{j, i_{a}} \cdot x_{i_{a}}\right\| \text { s.t. } W_{j, i_{a}} \gg 0, i_{a} \in \mathcal{N}\left(x_{j}\right) ; 1 \leq a \leq t .
$$


From Equation (1), a linear least square issue is fitted to optimize the weight vector, which can be found simply via a quadratic programming solver. Then, the row $\mathbf{W}=\mathbf{D}^{(-1)} \mathbf{W}$ is utilized to normalize weight matrix $\mathbf{W}$. Here, $\mathbf{D}=\operatorname{diag}$ $\left[d_{1}, d_{2}, \hat{\mathrm{a}}\right]$ is a diagonal matrix with $d_{j}=\sum_{(i=1)}^{s} w_{(j, i)}$.

3.4. Iterative Label Propagation Algorithm. Four normalized weight matrices are established for facilitating the iterative label propagation step in the label propagation step separately. Particularly, $\mathbf{H}=\operatorname{WGC}\left(\mathscr{C}_{p}, \mathscr{C}_{u}, k\right)$ is utilized for the label propagation from source domain set $\mathscr{C}_{p}$ to target domain set $\mathscr{C}_{u} . \mathbf{J}=\operatorname{WGC}\left(\mathscr{C}_{p}, \mathscr{C}_{p}, k\right)$ is proposed for the label propagation from $\mathscr{C}_{p}$ to itself. $\mathbf{V}=W G C\left(\mathscr{C}_{u}, \mathscr{C}_{u}, k\right)$ is utilized for the label propagation from $\mathscr{C}_{u}$ to itself. $\mathbf{L}=W G C\left(\mathscr{C}_{u}, \mathscr{C}_{p}, k\right)$ is constructed for the label propagation from $\mathscr{C}_{u}$ to $\mathscr{C}_{p}$. Detailed description of the algorithm is illustrated in reference [6].

\section{Experimental Validation}

In this work, to prove the proposed method, the compared methods is introduced. These approaches contains: GFK [30] (geodesic flow kernel), TJM [31] (transfer joint matching), JDA [32] (joint distribution adaptation), and TCA [33] (transfer component analysis).

4.1. Dataset. By Case Western Reserve University [34] and Guilin University of Electronic Technology, the datasets are acquired. The used data were obtained by the $0 \mathrm{HP}, 1$ HP, $2 \mathrm{HP}$, and $3 \mathrm{HP}$. The four fault classes concludes outer race fault (OF), inner race fault (IF), ball fault (BF), and normal (N). Therefore, 12 transfer fault experiments have been used to validate the proposed method in this section in Figure 3. In addition, the three faults' diameters were $0.007,0.014$, and 0.021 inches, respectively. Therefore, ten conditions under four loads are used to demonstrate the performance of the proposed method. Parameters applied by the proposed method are set as $k=10, \alpha=0.70$, $\beta=0.25, r=0.7$, and $T=100$. They are described as follows.

In this section, the proposed method is proved in 12 transfer experiments, which contains $\mathrm{TC}_{01}, \mathrm{TC}_{02}, \mathrm{TC}_{03}, \mathrm{~T}$ $\mathrm{C}_{10}, \mathrm{TC}_{12}, \mathrm{TC}_{13}, \mathrm{TC}_{20}, \mathrm{TC}_{21}, \mathrm{TC}_{23}, \mathrm{TC}_{30}, \mathrm{TC}_{31}$, and $\mathrm{TC}_{32}$. The $\mathrm{TC}_{01}$ represents the $0 \mathrm{HP}$ in the source domain and 1 HP in the target domain. The labeled samples are acquired in each transfer task of the source domain, and the unlabeled samples are acquired in each transfer task of the target domain.

4.1.1. Transfer Fault Identify Results in CWRU Dataset. The labeled examples are obtained in the source domain, and the unlabeled samples are acquired in target domain. The partial label samples are obtained in the whole training instance by randomly sampling $p \in 0.10,0.20,0.30,0.40$. The experimental results are presented in Figure 4. As is illustrated in Figure 4, the results of five transfer fault detection experiments are shown. The transfer results of the proposed method are also compared with four approaches. According to the comparison results, it can be seen that the proposed

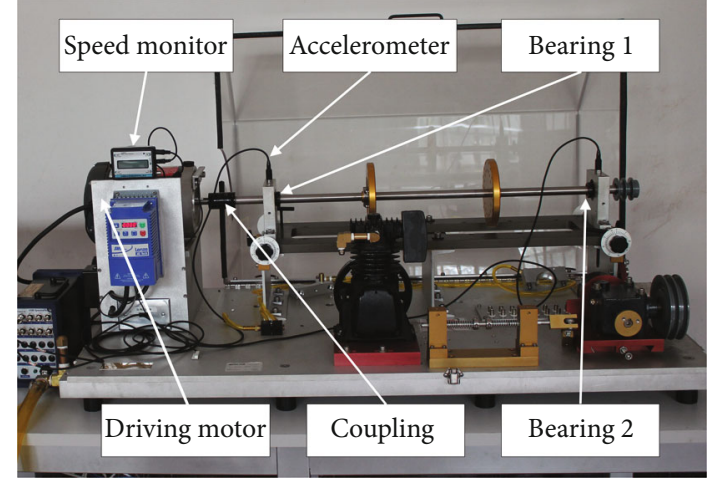

FIgURe 5: Machinery Fault Simulator test rig.

approach obtains the highest testing accuracy in the 12 transfer tasks among these four methods.

From Figure 4(a), the average testing accuracy of the proposed method is $95.6 \%$, which is the highest one among these four methods. Due to be unsuitable to deal with tensor data, the average testing accuracy of JDA reaches $35.7 \%$, which is smaller than the accuracy obtained by the proposed method. The average testing accuracy of TJM reaches 34.7\% and the accuracy of GFK is $31.8 \%$. Because they cannot extract high-level signatures from tensor samples of the target-domain, these two techniques realize poorer testing accuracies than the proposed method. In terms of TCA, its average accuracy reaches $37.8 \%$, which is smaller than the testing accuracy realized by the proposed method.

When $p=0.20$, the experimental results are presented in Figure 4(b). From Figure 4(b), the average testing accuracy of the proposed method is $96.8 \%$, which is the highest one among these four methods. Due to be unsuitable to deal with tensor data, the average accuracy of JDA is $37.3 \%$. The average testing accuracy of TJM reaches $36.8 \%$ and the accuracy of GFK is $33.9 \%$. In terms of TCA, its average accuracy reaches $39.7 \%$.

When $p=0.30$, the experimental results are presented in Figure 4(c). From Figure 4(c), the average testing accuracy of the proposed method is $97.9 \%$, which is the highest one among these four methods. Due to be unsuitable to deal with tensor data, the average accuracy of JDA is $39.2 \%$. The testing accuracy of TJM is $38.4 \%$, and the accuracy of GFK is $36.8 \%$. In terms of TCA, its average accuracy reaches $41.8 \%$.

The experimental results are presented in Figure 4(d) when $p=0.40$. From Figure $4(\mathrm{~d})$, the average testing accuracy of the proposed method is $98.9 \%$, which is the highest one among these four methods. Due to being unsuitable to deal with tensor data, the average testing accuracy of JDA reaches $41.4 \%$. The average testing accuracy of TJM reaches $41.9 \%$, and the accuracy of GFK is $39.4 \%$. In terms of TCA, its average accuracy reaches $44.8 \%$.

According to the experimental results, the presented technique can correctly and accurately classify the 12 transfer tasks in the target domain. The results clearly show that TRSSPLL technology can identify fault categories more accurately and effectively than other methods.

4.1.2. Transfer Fault Identify Results in GUET Dataset. In this section, the data is acquired by Guilin University of 
(a)

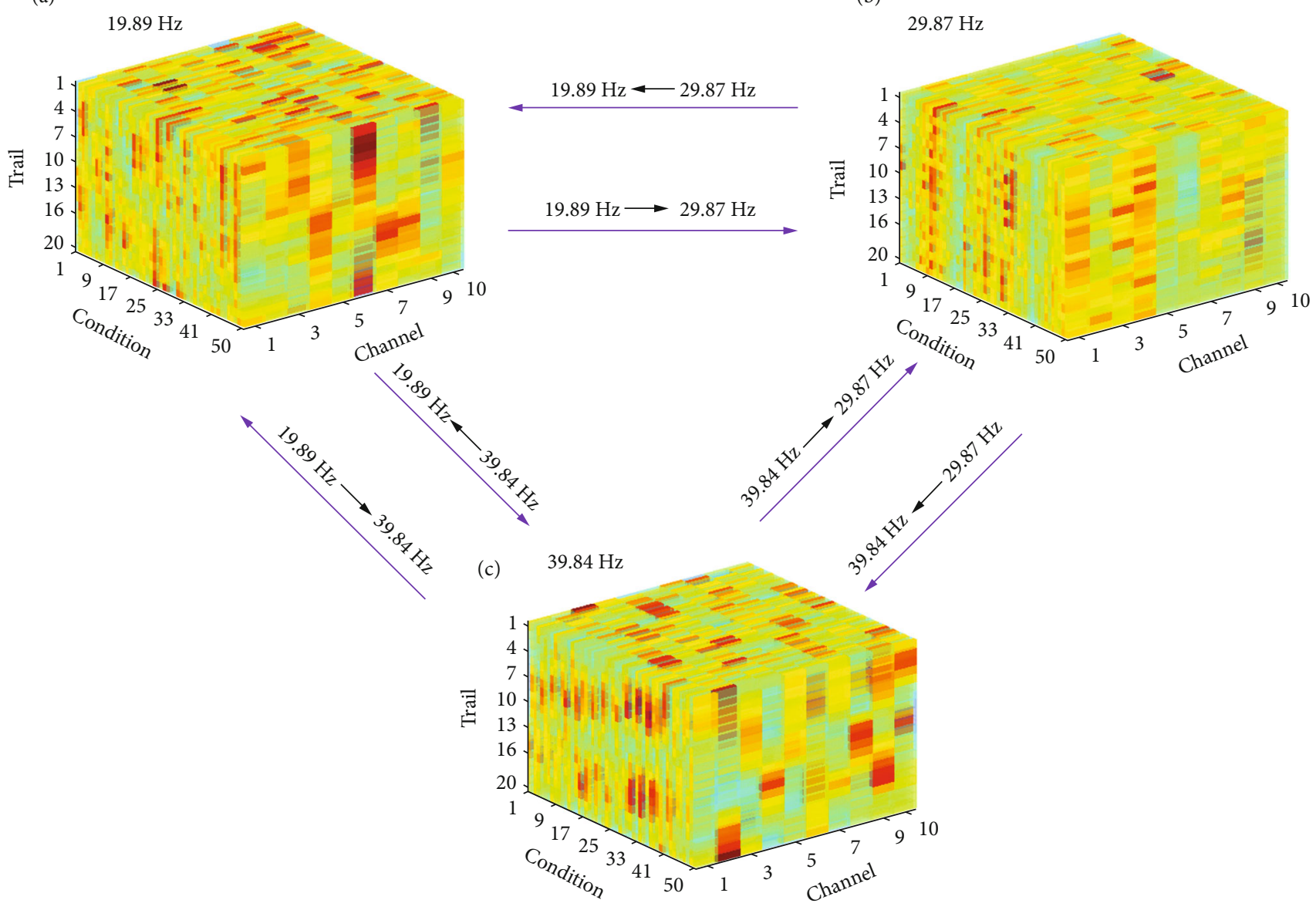

Figure 6: The 6 transfer fault experiments of three rotating frequency schemes: (a) $19.89 \mathrm{~Hz}$, (b) $29.87 \mathrm{~Hz}$, and (c) $39.84 \mathrm{~Hz}$.

Electronic Technology (GUET) for validating the presented technique. Figure 5 contains outer race fault $(\mathrm{OF})$, ball fault (BF), inner race fault (IF), and normal (N). Used transfer data were obtained with three rotating frequency $19.89 \mathrm{~Hz}$, $29.87 \mathrm{~Hz}$, and $39.84 \mathrm{~Hz}$. Therefore, four gear conditions under three rotating frequency schemes are established to validate performance of the proposed method in Figure 6. The DA transfer task of $\mathrm{TC}_{12}$ is selected in this work. The partial label examples are obtained in the whole training instance by randomly sampling $p \in 0.10,0.20,0.30,0.40$.

The experimental results are showed in Figure 7. Compared with the other approaches, the presented approach can realize the best result. This further demonstates effectiveness and superiority of the proposed method. In addition, transfer diagnosis tasks can benefit from DA algorithms. The GUET data demonstrate the performance of the presented approach.

When $p=0.10$, the results of the six cross-domain fault detection methods are shown in Figure 7(a). From Figure 7(a), it can be seen that the average test accuracy of the proposed algorithm reaches $96.4 \%$, which is the highest of the five methods. The average test accuracy of JDA, TJM, GFK, and TCA reached $35.7 \%, 43 \%, 49 \%$, and $44.2 \%$, respectively.

When $p=0.20$, the results of the six cross-domain fault detection methods are shown in Figure 7(b). It can be seen that the average test accuracy of the proposed algorithm reaches $97.9 \%$, which is the highest of the five methods. The average test accuracy of JDA, TJM, GFK, and TCA reached $37.5 \%, 45 \%, 507 \%$, and $46.4 \%$, respectively.

When $p=0.30$, the results of the six transfer fault diagnosis methods are shown in Figure 7(c). The average accuracy of proposed algorithm is $98.6 \%$, which is the highest of the five methods. The average test accuracy of JDA, TJM, GFK, and TCA reached 39.5\%, 47.4\%, 52.8\%, and $48.6 \%$, respectively.

When $p=0.40$, the results of the six cross-domain fault detection methods are shown in Figure $7(\mathrm{~d})$. The average test accuracy of the proposed algorithm reaches $99.4 \%$, which is the highest of the five methods. The average test accuracy of JDA, TJM, GFK, and TCA reached $41.6 \%$, $49.3 \%, 54.6 \%$, and $50.8 \%$, respectively.

According to the experimental results, the presented approach can accurately identify the six transfer tasks in the target domain. The results clearly show that the proposed technology can identify fault categories more accurately and effectively than other methods.

The proposed method is validated by different experiment data. The purpose of this paper is to deal with tensor data in source and target domain. As for JDA, TJM, GFK, and TCA, they are traditional transfer algorithms. It is 


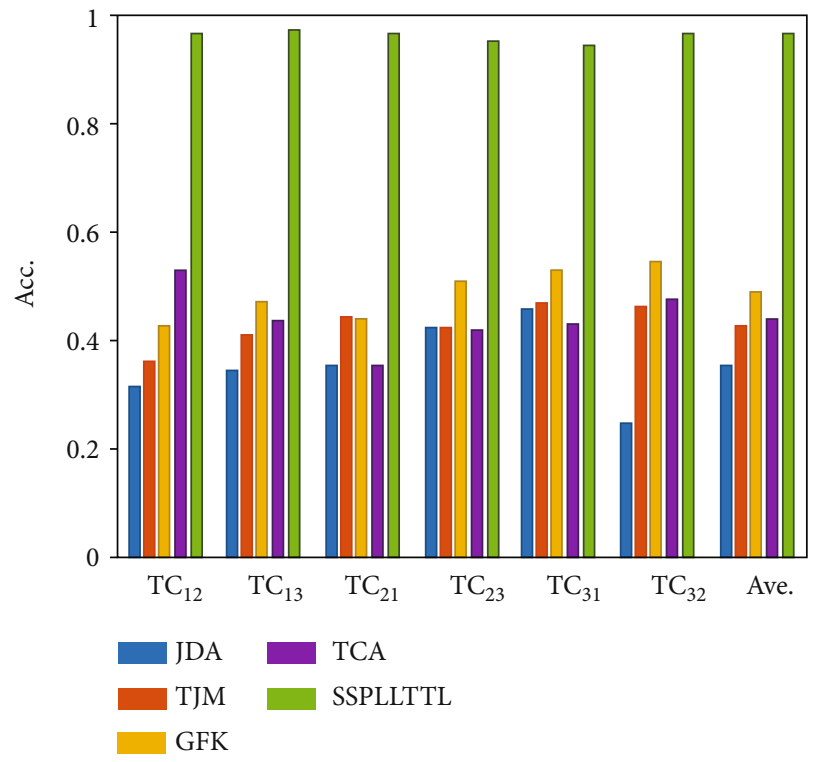

(a)

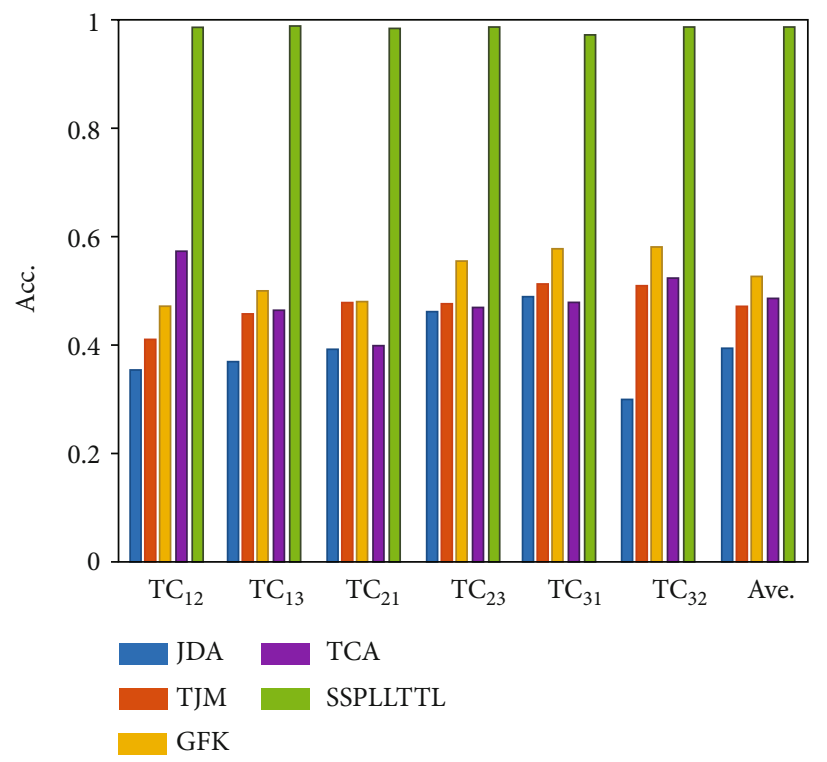

(c)

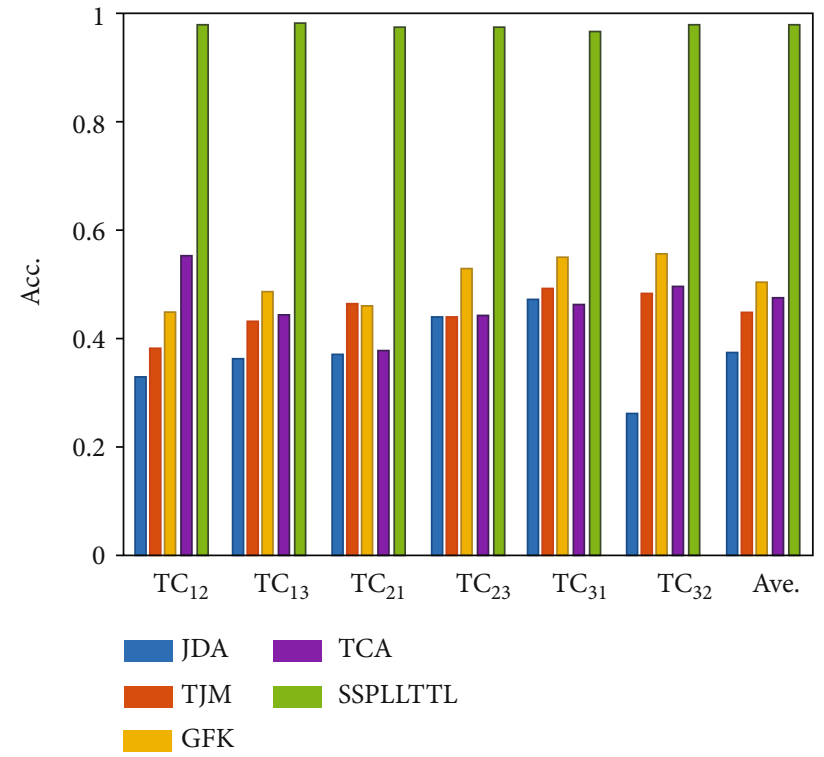

(b)

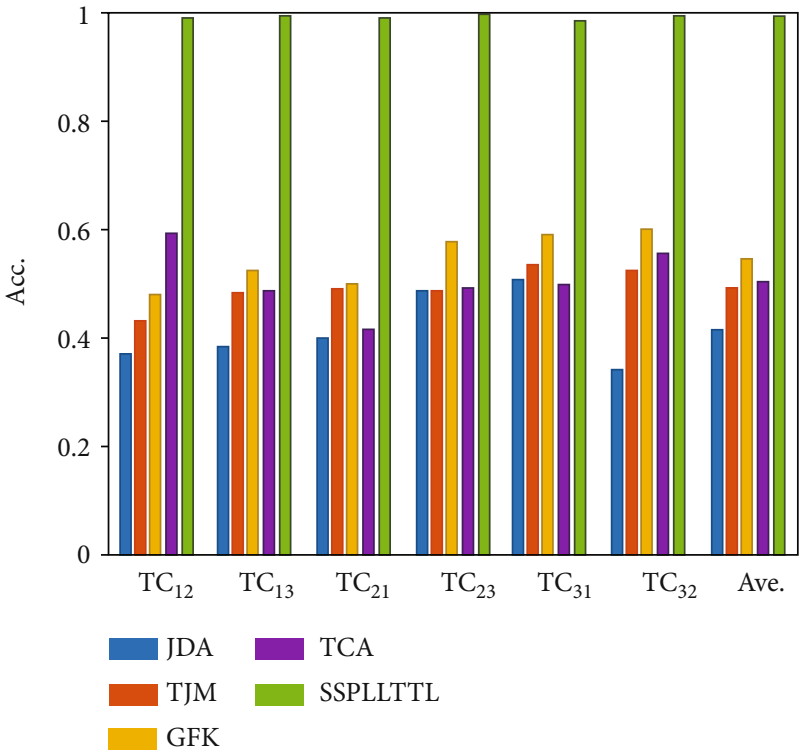

(d)

FIGURE 7: The partial label examples are obtained in the whole training instance by randomly sampling: (a) $p=0.1$, (b) $p=0.2$, (c) $p=0.3$, and (d) $p=0.4$.

undeniable that they have many advantages. They can only tackle vector-based data with cross-domain issue.

4.1.3. Comparisons with Related Works. General fault identify issues assume the training and testing data are obtained from the same machine.Various fault classification methods [35-38] have realized very high testing accuracy. However, these methods can not deal with the cross-domain fault diagnosis issue. For the transfer fault diagnosis issue, which the source and the target domains data are acquired for training model. In existing literature, the transfer fault diagnosis methods $[17,39]$ are proposed for tackling domain shift problems. Nevertheless, the proposed approach can not handle with tensor data. Aside from this, the unlabeled data is not considered by these fault diagnosis techniques. Thus, for dealing with transfer, tensor data issues, and unlabeled data, a new tensor transfer approach is proposed for rotating machinery intelligent fault diagnosis with semisupervised partial label learning in this paper.

In this paper, the data of training and testing are acquired in source and the target domains separately, so the transfer fault detection experiment is more difficult than existing cross-domain task. The testing accuracy has been realized $98.9 \%$ and $99.4 \%$ in two transfer tasks by the promoted technique, respectively. Therfore, according to consequences, the proposed method are competitive. 


\section{Conclusion}

Since some information is hard to denote by vector arithmetic, thus, a new DA method based on tensor representation is first applied to adapt the source and target domains tensor data directly, without vectorization in the field of intelligent fault diagnosis. Then, SSPLL is proposed for training set consists of two kinds of weak supervision, i.e., partial label data and unlabeled data. An iterative label propagation method is introduced, which can process two kinds of weakly supervised data simultaneously by jointly propagating label between partial labeled and unlabeled instances and derive a good label assignment.

The employed approach realizes higher classification accuracy of bearing heath states compared with vectorbased representation algorithms. Aside from this, the experimental results verify that the presented approach is superior to methods that only considering one kind of weak supervision. In future works, the model will be used to large-scale data, and weak supervision data will also be considered in dynamic environments. Then, a powerful invariant tensor subspace need to be learned in further research works.

\section{Data Availability}

The data used to support the findings of this study can be obtained through the first author's email.

\section{Conflicts of Interest}

The authors declare that they have no conflicts of interest.

\section{Acknowledgments}

This study is supported by the subject of Director of Guangxi Key Laboratory of Manufacturing System and Advanced Manufacturing Technology, Guilin University of Electronic Technology (Grant no. 20-065-40-004Z), Natural Science Foundation of Guangxi (Grant no. 2021GXNSFBA075050), Middle-aged and Young Teachers' Basic Ability Promotion Project of Guangxi (Grant no. 2021KY0213), National Natural Science Foundation of China (Grant no. 52065013 and 51965013), and Natural Science Foundation of Guangxi, China (Grant nos. 2018GXNSFAA281276 and 2020GXNSFAA159081).

\section{References}

[1] Z. He, H. Shao, Z. Ding, H. Jiang, and J. Cheng, "Modified deep auto-encoder driven by multi-source parameters for fault transfer prognosis of aero-engine," IEEE Transactions on Industrial Electronics, no. 99, 2021.

[2] Q. Zhu, B. Sun, Y. Zhou, W. Sun, and J. Xiang, "Sample augmentation for intelligent milling tool wear condition monitoring using numerical simulation and generative adversarial network," IEEE Transactions on Instrumentation and Measurement, vol. 70, pp. 1-10, 2021.

[3] Y. Zhou, B. Sun, W. Sun, and Z. Lei, "Tool wear condition monitoring based on a two-layer angle kernel extreme learning machine using sound sensor for milling process," Journal of Intelligent Manufacturing, 2020.
[4] Z. Lei, Y. Zhou, B. Sun, and W. Sun, "An intrinsic timescale decomposition-based kernel extreme learning machine method to detect tool wear conditions in the milling process," The International Journal of Advanced Manufacturing Technology, vol. 106, no. 3-4, pp. 1203-1212, 2020.

[5] C. Hu and Y. Wang, "Multidimensional denoising of rotating machine based on tensor factorization," Mechanical Systems and Signal Processing, vol. 122, pp. 273-289, 2019.

[6] Q. W. Wang, Y. F. Li, and Z. H. Zhou, "Partial label learning with unlabeled data," in Proceedings of the 28th International Joint Conference on Artificial Intelligence, pp. 3755-3761, Macao, China, 2019.

[7] X. Liu, H. Huang, and J. Xiang, "A personalized diagnosis method to detect faults in a bearing based on acceleration sensors and an fem simulation driving support vector machine," Sensors, vol. 20, no. 2, p. 420, 2020.

[8] W. Qian, S. Li, J. Wang, and Q. Wu, "A novel supervised sparse feature extraction method and its application on rotating machine fault diagnosis," Neurocomputing, vol. 320, pp. 129140, 2018.

[9] X. Zhao and M. Jia, "A new local-global deep neural network and its application in rotating machinery fault diagnosis," Neurocomputing, vol. 366, pp. 215-233, 2019.

[10] K. Xu, S. Li, X. Jiang, Z. An, J. Wang, and T. Yu, “A renewable fusion fault diagnosis network for the variable speed conditions under unbalanced samples," Neurocomputing, vol. 379, pp. 12-29, 2020.

[11] H. Shao, M. Xia, J. Wan, and C. de Silva, "Modified stacked auto-encoder using adaptive Morlet wavelet for intelligent fault diagnosis of rotating machinery," IEEE/ASME Transactions on Mechatronics, no. 99, p. 1, 2021.

[12] X. Liu, H. Huang, and J. Xiang, "A personalized diagnosis method to detect faults in gears using numerical simulation and extreme learning machine," Knowledge Based Systems, vol. 195, article 105653, 2020.

[13] Y. Gao, X. Liu, and J. Xiang, "Fem simulation- based generative adversarial networks to detect bearing faults," IEEE Transactions on Industrial Informatics, vol. 16, no. 7, pp. 4961-4971, 2020.

[14] Z. He, H. Shao, J. Cheng, X. Zhao, and Y. Yang, "Support tensor machine with dynamic penalty factors and its application to the fault diagnosis of rotating machinery with unbalanced data," Mechanical Systems and Signal Processing, vol. 141, article 106441, 2020.

[15] C. Hu, Y. Wang, and T. Bai, "A tensor-based approach for Identification of multi-channel bearing compound faults," IEEE Access, vol. 7, pp. 38213-38223, 2019.

[16] C. Hu and Y. Wang, "Research on multi-channel signal denoising method for multiple faults diagnosis of rolling element bearings based on tensor factorization," Journal of Mechanical Engineering, no. 12, p. 7, 2019.

[17] X. Li, W. Zhang, and Q. Ding, “Cross-domain fault diagnosis of rolling element bearings using deep generative neural networks," IEEE Transactions on Industrial Electronics, vol. 66, no. 7, pp. 5525-5534, 2019.

[18] L. Guo, Y. Lei, S. Xing, T. Yan, and N. Li, "Deep convolutional transfer learning network: a new method for intelligent fault diagnosis of machines with unlabeled data," IEEE Transactions on Industrial Electronics, vol. 66, no. 9, pp. 7316-7325, 2019.

[19] H. Lu, L. Zhang, Z. Cao et al., "When unsupervised domain adaptation meets tensor representations," in 2017 IEEE 
International Conference on Computer Vision (ICCV), pp. 599-608, Venice, Italy, 2017.

[20] R. Aljundi, R. Emonet, D. Muselet, and M. Sebban, "Landmarks-based kernelized subspace alignment for unsupervised domain adaptation," in Proceedings of the IEEE Conference on Computer Vision and Pattern Recognition, pp. 56-63, Boston, MA, USA, 2015.

[21] B. Fernando, A. Habrard, M. Sebban, and T. Tuytelaars, "Unsupervised visual domain adaptation using subspace alignment," in 2013 IEEE International Conference on Computer Vision, pp. 2960-2967, Sydney, NSW, Australia, 2013.

[22] Y. Yu, H. Cao, X. Yan, T. Wang, and S. S. Ge, "Defect identification of wind turbine blades based on defect semantic features with transfer feature extractor," Neurocomputing, vol. 376, pp. 1-9, 2020.

[23] Z. Zhang, H. Chen, S. Li, Z. An, and J. Wang, "A novel geodesic flow kernel based domain adaptation approach for intelligent fault diagnosis under varying working condition," Neurocomputing, vol. 376, pp. 54-64, 2020.

[24] B. Yang, Y. Lei, F. Jia, and S. Xing, "An intelligent fault diagnosis approach based on transfer learning from laboratory bearings to locomotive bearings," Mechanical Systems and Signal Processing, vol. 122, pp. 692-706, 2019.

[25] Z. Chen, K. Gryllias, and W. Li, "Intelligent fault diagnosis for rotary machinery using transferable convolutional neural network," IEEE Transactions on Industrial Informatics, vol. 16, no. 1, pp. 339-349, 2020.

[26] Y. C. Chen, V. M. Patel, R. Chellappa, and P. J. Phillips, "Ambiguously labeled learning using dictionaries," IEEE Transactions on Information Forensics and Security, vol. 9, no. 12, pp. 2076-2088, 2014.

[27] M. L. Zhang and F. Yu, "Solving the partial label learning problem: an instance-based approach," in Twenty-Fourth International Joint Conference on Artificial Intelligence, Buenos Aires, Argentina, 2015.

[28] C. Z. Tang and M. L. Zhang, "Confidence-rated discriminative partial label learning," in Thirty-first AAAI Conference on artificial Intelligence, San Francisco California USA, 2017.

[29] G. J. Hu Chaofan and W. Yanxue, "Cross-domain intelligent fault classification of bearings based on tensor-aligned invariant subspace learning and two-dimensional convolutional neural networks," Knowledge-Based Systems, 2020.

[30] B. Gong, Y. Shi, F. Sha, and K. Grauman, "Geodesic flow kernel for unsupervised domain adaptation," in 2012 IEEE Conference on Computer Vision and Pattern Recognition, pp. 20662073, Providence, RI, USA, 2012.

[31] M. Long, J. Wang, G. Ding, J. Sun, and P. S. Yu, “Transfer joint matching for unsupervised domain adaptation," in Proceedings of the IEEE conference on computer vision and pattern recognition, pp. 1410-1417, Columbus, OH, USA, 2014.

[32] M. Long, J. Wang, G. Ding, J. Sun, and P. S. Yu, "Transfer feature learning with joint distribution adaptation," in Proceedings of the IEEE international conference on computer vision, pp. 2200-2207, Sydney, NSW, Australia, 2013.

[33] S. J. Pan, I. W. Tsang, J. T. Kwok, and Q. Yang, "Domain adaptation via transfer component analysis," IEEE Transactions on Neural Networks, vol. 22, no. 2, pp. 199-210, 2011.

[34] W. A. Smith and R. B. Randall, "Rolling element bearing diagnostics using the case western reserve university data: a benchmark study," Mechanical Systems and Signal Processing, vol. 64-65, pp. 100-131, 2015.
[35] Z. X. Hu, Y. Wang, M. F. Ge, and J. Liu, "Data-driven fault diagnosis method based on compressed sensing and improved multiscale network," IEEE Transactions on Industrial Electronics, vol. 67, no. 4, pp. 3216-3225, 2020.

[36] Y. Yang, H. Zheng, Y. Li, M. Xu, and Y. Chen, "A fault diagnosis scheme for rotating machinery using hierarchical symbolic analysis and convolutional neural network," ISA Transactions, vol. 91, pp. 235-252, 2019.

[37] J. Liang, Y. Zhang, J.-H. Zhong, and H. Yang, "A novel multisegment feature fusion based fault classification approach for rotating machinery," Mechanical Systems and Signal Processing, vol. 122, pp. 19-41, 2019.

[38] X. Yan and M. Jia, "Intelligent fault diagnosis of rotating machinery using improved multiscale dispersion entropy and mrmr feature selection," Knowledge-Based Systems, vol. 163, pp. 450-471, 2019.

[39] H. Zheng, R. Wang, J. Yin, Y. Li, H. Lu, and M. Xu, “A new intelligent fault identification method based on transfer locality preserving projection for actual diagnosis scenario of rotating machinery," Mechanical Systems and Signal Processing, vol. 135, article 106344, 2020. 